\title{
Modality and Paradox
}

\author{
December 30, 2014
}

\begin{abstract}
Philosophers often explain what could be the case in terms of what is, in fact, the case at one possible world or another. They may differ in what they take possible worlds to be or in their gloss of what is for something to be the case at a possible world. Still, they stand united by the threat of paradox. A family of paradoxes akin to the set-theoretic antinomies seem to allow one to derive a contradiction from apparently plausible principles. Some of them concern the interaction between propositions and worlds, and they appear to afford the means to map classes of propositions into propositions-or, likewise, classes of worlds into worlds-in a one-to-one fashion that leads to contradiction. Yet another family of paradoxes threaten the view that whatever could exist does, in fact, exist, which is in line with modal realism, for example. This article aims to survey and identify the source of each family of paradoxes as well as to outline some responses to them.
\end{abstract}

\section{Introduction}

We often contrast what could merely the case with what is, in fact, the case. It is possible that the election could have had a different outcoume. But what exactly is for this possibility to obtain? Possible worlds accounts of modality explain the fact that the election could have had a different outcome in terms of the fact that the election does, in fact, have a different outcome at some possible worlds. More generally, they take a proposition to be possible if, and only if, it is true at some possible world:

$(\diamond)$ It is possible that $p$ if, and only if, the proposition that $p$ is true at some possible world $w$.

Notice that the schema remains silent as to what is the nature of possible worlds or what is the best way to understand what is for a proposition to be true at a possible world. Modal realism, as developed by (Lewis 1986), takes possible worlds to be causally and spatiotemporally isolated concrete universes. So, if it is possible that the election could have had a different outcome, then there is a concrete universe, which is causally and spatiotemporally isolated from us at which 
we find the election to have a different outcome. However, the modal realist interpretation of possible worlds talk is far from compulsory. Other philosophers have identified possible worlds with states of affairs, (Plantinga 1976) and (Plantinga 1992)), propositions and classes thereof, ((Adams 1974)), possible states of the universe (Kripke 1980), and ways the universe might have been (Stalnaker 1976). ${ }^{1}$

But whatever possible worlds may be, propositions are said to be true at them, and apparently plausible principles concerned with the interaction between worlds and propositions are prone to paradox. In this article, we survey two common examples. One paradox is a direct descendant of Russell's paradox of propositions, and it is premised on the existence of a one-one map from classes of propositions into propositions. If there are possible worlds and propositions are true at them, then each world should determine a class of propositions which contains, for each proposition, either it or its negation. A Russell-style argument is supposed to lead to contradiction. The other paradox seeks to derive the existence of a one-one map from classes of possible worlds into possible worlds from eminently reasonable assumptions. And we will again be led to contradiction unless we find a way to resist the existence of such a map.

A different family of paradoxes targets the view that whatever could exist does, in fact, exist. While this claim is not forced upon one by $(\diamond)$, it fits well with modal realism: when we open the range of our quantifiers to vary over all there is, we find that they range over a plethora of merely possible objects as well as actual ones. We will explore ways in which such an expansive ontology is prone to paradox.

Before we survey each family of problems, we introduce three themes that will recur across the formulation of different difficulties.

\section{The Toolkit}

First, three set-theoretic preliminaries. One concerns the alleged distinction between sets and classes, another is a general device for cardinality comparisons, and the last one concerns applications of set theory in non-mathematical contexts.

\subsection{Sets and classes}

One of the morals of the set-theoretic antinomies is that there is no set of all non-self-membered sets. And yet, we find it almost irresistible to speak of the collection of non-self-membered sets.

\footnotetext{
${ }^{1}$ See (deRosset 2009a) and (deRosset 2009b) for a survey of possible accounts of modality.
} 
The distinction between sets and classes is supposed to accommodate such talk. Sets and classes are sometimes conceived as collections, but some classes are collections that are too large to form a set, e.g., the class of all non-self-membered sets; they are called 'proper classes'. Like sets, classes are extensional: if two classes have different members, then they are different. But, unlike sets, classes are never members.

Classes, when conceived as set-like objects other than sets, are a source of perplexity: sets have elements and classes have members; sets are themselves elements of sets and members of classes, but classes themselves are never members of anything. One question at this point is what exactly bars the formation of further collections of classes. And a second question is how exactly to square the existence of proper classes with the thought that set theory is the most comprehensive theory of collections.

On the other hand, classes have their uses in set theory: they allow one to turn some axiom schemata - such as separation and replacement-into single axioms; they play a crucial role in the motivation and formulation of certain large cardinal axioms; and they are often invoked in settheoretic argumentation. ${ }^{2}$ Fortunately, there is no need to take talk of classes at face value in order to enjoy the benefits they bring with them.

Set theorists often prefer to paraphrase talk of classes in terms of satisfaction, and they take a set to be a member of the class if, and only if, it satisfies an open formula of the language of set theory. Unfortunately, this interpretation is not sufficiently general to accommodate all uses to which classes are put in set theory, though it is all we need to show that a class has strictly more subclasses than members. ${ }^{3}$ Alternatively, we may understand singular quantification over classes as shorthand for plural quantification over their members; to speak of a class of all possible worlds is to speak of all possible worlds in the plural, and to claim that a given world is a member of a given class of worlds is to claim that it is one of them.

In what follows, we opt for the plural interpretation of class talk, which is sufficiently general for the purposes of this paper. While the metalinguistic interpretation is enough to underwrite Kaplan's paradox, for example, it turns out to be inadequate to make sense of Russell's paradox of propositions.

\footnotetext{
${ }^{2}$ See (Maddy 1983) for a classic discussion of the role of classes in set theory.

${ }^{3}$ In particular, it cannot accommodate impredicative classes specified by appeal to quantification over classes, even though they play a role in the motivation of certain large cardinal axioms.
} 


\subsection{Cantor's theorem}

We will be concerned with cardinality comparisons between collections of propositions and propositions, and, likewise, comparisons between collections of worlds and worlds. So, let us begin with the question of how to compare the cardinality of two sets. In set theory, comparisons of cardinality involve the existence of functions from one set to another. For example, two sets $a$ and $b$ are called equinumerous if, and only if, there is a bijection between $a$ and $b$. And a set $a$ is less numerous than another set $b$ if, and only if, there is a one-one function from $a$ into $b$, but not vice versa. ${ }^{4}$

Cantor's theorem is often glossed as the observation that a set has strictly more subsets than elements. Given our definitions, this is tantamount to the claim that there is a one-one function from $a$ into $\mathcal{P} a$ but not vice versa. We know of course that there is a one-one function $f$ from $a$ into $\mathcal{P} a$, where $f(a)=\{a\}$. But there is no one-one function from $\mathcal{P} a$ into $a$; otherwise, there would be a function from $a$ onto $\mathcal{P} a$, which is impossible. ${ }^{5}$

- Cantor's theorem: If $f$ is a function from a given set $a$ to $\mathcal{P} a$, then $f$ is not onto.

Assume $f$ is a function from $a$ to $\mathcal{P} a$. Let $d$ be a subset of $a$ given by: $\{x \in a: x \notin f(x)$, which exists by the axiom of separation. $d$ is not the image of any element of $a$ under $f$ : if $f(c)=d$, for some $c \in a$, then $c \in d$ iff $c \notin f(c)$ iff $c \notin d$, which leads to contradiction. It follows that $f$ is not onto.

The proof of Cantor's theorem takes place in Zermelo-Fraenkel set theory (ZF) but breaks down in alternative set theories with a universal set such as, for example, Quine's New Foundations (NF). ${ }^{6}$ Because NF allows for the existence of a universal set $U$ of all sets, it is not difficult to check that $U$ has no more subsets than it has elements. We lack the space to discuss NF and related systems,

${ }^{4}$ Some definitions:

- A function $f$ is a set of ordered pairs such that for all $x, y, z,\langle x, y\rangle \in f$ and $\langle x, z\rangle \in f$, then $y=z$. We write $f(x)=y$ to abbreviate: $\langle x, y\rangle \in f$.

- $f$ is a function from a to $b$ iff the domain of $f$, i.e., $\{x: \exists y\langle x, y\rangle \in f\}$, is $a$ and the range of $f$, i.e., $\{x: \exists y\langle y, x\rangle \in f\}$ is a subset of $b$.

- $f$ is a function from $a$ onto $b$ iff $f$ is a function from $a$ to $b$ and the range of $f$ is identical to $b$.

- $f$ is a one-one function from $a$ into $b$ iff $f$ is a function from $a$ to $b$ and forall $x, y$ in the domain of $f, f(x)=f(y)$ only if $x=y$.

- $f$ is a bijection between $a$ and $b$ iff $f$ is a one-one function from $a$ onto $b$.

${ }^{5}$ If $f$ is a one-one function from $a$ into $b$, then the inverse function $f^{-1}$ would be a function from $b$ onto $a$. The inverse function, $f^{-1}$, of $f$ is defined: $f^{-1}(y)=x$ iff $f(x)=y$.

${ }^{6}$ Quine proposed NF in Quine 1937, and no contradictions are known to follow from it. However, the axiom of choice is known to fail in it. 
but it should perhaps be noted that some philosophers have recently turned to Quine's framework in order to seek refuge from some of the problems to be discussed below. Three recent examples of this strategy are (Oksanen 1999), (Cocchiarella 2000), and (Cantini 2004).

In standard set theory, Cantor's theorem settles the question of whether a set has more subsets than elements, but it does not speak to the question of whether a class has more subclasses than members. There is, however, a generalization of Cantor's theorem according to which a class has indeed strictly more subclasses than members. One difficulty at this point is that given a class $A$, there is no sense to be made of the class of all subclasses of $A$, since, unlike sets, classes themselves are never members. Moreover, even if we generalize the definition of a function to allow for certain classes of ordered pairs to count as functions, there is still no sense to be made of a function from $A$ to subclasses of $A .^{7}$ The problem, in short, is that we seem to lack the resources to make sense of a class-valued function, one which assigns classes to sets.

(Bernays 1942) explained how to code a class-valued function by means of a relation, which pairs a member of the domain with every member of the class we choose to assign to it. Now, a relation is just a class of ordered pairs, and a class-valued function from a given class $A$ to subclasses of $A$ becomes a relation that pairs a member $a$ of $A$ with each member $b$ of the class $B$ assigned to $a$. We may even write $R(b)=B$ to abbreviate: $\forall x(\langle b, x\rangle \in R \leftrightarrow x \in B)$.

Bernays' generalization of Cantor's theorem for classes is the observation that no class-valued function from a given class $A$ onto subclasses of $A$ will be onto: given such a class-valued function, there is, on pain of contradiction, a subclass $D$ of $A$ not assigned to any member of $A$.

- Bernays' theorem: If $R$ is a class-valued function from a given class $A$ to subclasses of $A$, then $R$ is not onto.

Assume $R$ is a class-valued function from $A$ to subclasses of $A$. Let $D$ be a subclass of $A$ given by: $\{x \in a:\langle x, x\rangle \in R\}$, which exists by an axiom of predicative class comprehension. ${ }^{8}$ Now, $D$ is not the image of any element of $A$ under $R$ : if $R(d)=D$, for some $d \in A$, then $\langle d, d\rangle \in R$ iff $d \in D$ iff $d \notin R(d)$ iff $\langle d, d\rangle \notin R$, which leads to contradiction. We conclude that $R$ is not onto.

By way of illustration, Bernays' theorem tells us that even if there is a proper class of possible worlds, there are still strictly more classes of possible worlds than there are possible worlds. If

\footnotetext{
${ }^{7}$ A functional class $F$ is a class of ordered pairs such that for all $x, y, z,\langle x, y\rangle \in F$ and $\langle x, z\rangle \in F$, then $y=z$. We write $F(x)=y$ to abbreviate: $\langle x, y\rangle \in f$. The definitions given in footnote 4 can be generalized for functional classes in a straightforward way.

${ }^{8}$ The axiom schema of predicative comprehension generates the existence of a class of members satisfying an open formula $A(x)$ of the language of class theory without any bound class variables.
} 
every class of possible worlds determine at least one proposition, then, by Bernays' theorem, there are strictly more propositions than there are possible worlds.

\subsection{Impure sets and classes}

Cantor's theorem, and Bernays' generalization, take place in a formal framework in which all quantification ranges over sets and classes, never individuals. In the absence of individuals, all elements of a set are sets, all elements of their elements are sets, etc: sets are pure, and classes contain only pure sets as members. In what follows, however, we will look at contexts in which quantification over individuals is not only permitted, but required. We will draw a contrast between individuals, which are elements but never contain elements, and sets and classes. Individuals are called ur-elements, and we will make allowance for impure sets and classes. ${ }^{9}$

Do the ur-elements form a set? Suppose that possible worlds, for example, are ur-elements. Then, if there is no set of them, then there is a proper class of ur-elements. This, however, may be thought to be in tension with the iterative conception of set. On the iterative conception, sets are formed in stages. Stage 0 consists of all ur-elements like chairs, tables, and possible worlds, and at stage 1 , we form all sets of ur-elements; in general, at a successor stage, we form all sets of sets formed at the immediately preceding stage; and at a limit stage, we form sets of sets formed at preceding stages of the iteration.

The iterative conception explains why there is no set of all sets: sets are formed at stages, but there is no stage in the iteration at which all sets are ever available for collection. So, there is never occasion to form a set of all sets. Unfortunately, no such iterative explanation is available when it comes to the question of why there is no set of all ur-elements. They are, after all, available at the very first stage of the cumulative hierarchy. So, it is not uncommon to take the iterative conception of set to motivate the hypothesis that the ur-elements form a set:

The Ur-element Set Axiom: There is a set of all ur-elements.

In Zermelo-Fraenkel set theory with choice (ZFC), this requires the ur-elements to be few in number and to be measured by some Cantorian cardinal number. But (Menzel 2014) has recently explored a modification of ZFCU intended to accommodate the existence of "wide sets", which, while formed at low stages of the cumulative hierarchy, are not "mathematically determinable". The axioms of replacement and power set, in particular, are appropriately restricted in order to accommodate them. ${ }^{10}$

\footnotetext{
${ }^{9} \mathrm{~A}$ set will be impure if it is not pure, e.g., it contains ur-elements as elements, as elements of elements, etc.

${ }^{10}$ Replacement is restricted to the claim that, roughly, only the range of an operation $F$ on mathematically determinable sets determines a set. The axiom of power set is similarly stated as the claim that for every set $a$, there is a
} 
However, strictly speaking, the iterative conception only requires that sets be formed in stages; it does not mandate the formation of a set of all ur-elements. How could the ur-elements fail to form a set, you ask? In Zermelo-Fraenkel set theory with choice (ZFC), one may prove that every set is equinumerous with the set of predecessors of an ordinal, which suggests an alternative account of proper classes: proper classes are too numerous to be in one-one correspondence with the set of predecessors of an ordinal. ${ }^{11}$ One model for the failure of the class of possible worlds to form a set would be one in which they are indeed too numerous to be equinumerous with the set of predecessors of an ordinal. ${ }^{12}$

On this picture of the cumulative hierarchy, stage 0 consists of a proper class of ur-elements-at least as numerous as the class of ordinals. Call a collection set-sized if, and only if, it is equinumerous with the set of predecessors of an ordinal. At stage 1, we form a set of ur-elements for each set-sized collection of them-notice, in particular, that we will not have occasion to form a set of all ur-elements. In general, at successor stages, we form a set for each set-sized collection of sets formed at immediately prior stages. At limit stages, we form a set for each set-sized collection of sets formed at earlier stages. At the end of the day, all proper classes are too numerous to be equinumerous with the set of predecessors of an ordinal. ${ }^{13}$

We lay out two final reasonable hypotheses. One the the thought that if $A$ and $B$ are too classes, then they are comparable in size, i.e., one is at least as numerous as the other.

Cardinal Comparability: If $A$ and $B$ are two classes, then there is a one-to-one map from one into the other.

In the context of a theory of classes over ZFC, Cardinal Comparability is equivalent to the hypothesis that all proper classes are equinumerous. ${ }^{14}$

set which contains all and only the "mathematically determinable" subsets of $a$. The proposal requires two more axioms intended to enforce the existence of a partition of the universe into ranks and the thought that only mathematically determinable sets admit an increase in cardinality.

${ }^{11}$ Since a von Neumann ordinal is identical with the set of its predecessors, this could be put in terms of a one-one correspondence with a von Neumann ordinal.

${ }^{12}$ There are, in addition, precedents for the failure of ur-elements to form a set in (Barwise 1975) and (Faithful Representation in Set Theory with Atoms).

${ }^{13}$ For a formal model, let $\kappa$ is the first strongly inaccessible cardinal and let $U$ is a set of ur-elements. We write $\mathcal{P}^{<\kappa}(X)$ to refer to the set of all subsets of $X$ of cardinality strictly less than $\kappa$, we define:

$$
\begin{array}{rlr}
U_{0}^{>} & =U \\
U_{\alpha+1}^{>} & =U \cup \mathcal{P}^{<\kappa}\left(U_{\alpha}\right) & \\
U_{\lambda}^{>} & =\bigcup_{\alpha<\lambda} U_{\alpha}, & \text { for } \lambda \text { a limit ordinal. }
\end{array}
$$

This construction yields a model of second-order ZFC with ur-elements on which there is no set of ur-elements.

${ }^{14}$ This is a special case of the result stated below in which we start with an empty class of ur-elements. 
Maximality: All proper classes are equinumerous.

Matters change a little when we allow for ur-elements. Maximality does not follow from Cardinal Comparability in the context of a theory of classes over Zermelo-Fraenkel set theory with choice and ur-elements (ZFCU), unless we assume that there are at least as many ordinals as there are ur-elements. ${ }^{15}$

\section{Possible worlds and paradox}

We now discuss two general threats for $(\diamond)$. One is a descendant of Russell's paradox of propositions, while the other has come to be known as Kaplan's paradox. Russell-style arguments are quite general, but they are open to the same response as Russell's original argument. Kaplan's paradox comes in at least two forms, and it requires quite a different response.

\subsection{Russell's Paradox of Propositions}

As developed in (Russell 1903), Russell's paradox of propositions involves three plausible principles:

$(R 1)$ For each class of propositions $C$, there is a proposition $p_{C}$, e.g., every proposition in $C$ is true, which is associated with it.

(R2) If $C$ and $D$ are two different classes of propositions, then $p_{C}$ is different from $p_{D}$.

(R3) There is a class of propositions $R$, which consists of all and only propositions $p$ such that $p=p_{C}$ for some class of propositions $C$ such that $p \notin C$.

Russell derived an outright contradiction from them:

If $R$ is the class of propositions described by $(R 3)$, let $p_{R}$ be the proposition associated with it, which exists by $(R 1)$. First, observe that $p_{R} \in R$ : otherwise, if $p_{R} \notin R$, then $p_{R}$ would be associated to a class to which it does not belong, namely, $R$, which would make $p_{R}$ a member of $R$ after all. Second, now that we know $p_{R} \in R$, note that, by definition of $R, p_{R}=p_{C}$ for some class $C$ to which $p_{R}$ does not belong. Since $p_{R} \in R$, $C \neq R$, which contradicts $(R 2)$.

Although Russell speaks of "classes of propositions", we need not assume that they form a proper class. If there is a set of all propositions, then $(R 1)$ and $(R 2)$ will already contravene Cantor's

\footnotetext{
${ }^{15}$ Similar textbook claims are discussed in (Rubin and Rubin 1985). (Uzquiano 2014) outlines a proof in the appendix.
} 
theorem, since they entail the existence of a one-one function from the power set of the set of all propositions and the set of all propositions, i.e., a one-one function which maps each and every set of propositions into a proposition. On the other hand, if there is a proper class of propositions, then Bernays' theorem provides another route to Russell's conclusion: $(R 1)$ and $(R 2)$ are true, then we should be able to specify a class-valued function, $R$, which pairs a class of propositions onto all classes of propositions. In particular, if $p_{C}$ is the proposition associated with the class $C$, then for all $q,\left\langle p_{C}, q\right\rangle \in R$ only if $q \in C .{ }^{16}$

What to do? It is not very plausible to deny $(R 1)$ : no matter what a class of propositions may be, we should be able to predicate something of it, e.g., that every one of its members is true. To avoid contradiction, we may attempt to reject one of $(R 2)$ or $(R 3)$.

Russell himself considered the first option. He noticed that $(R 2)$ collapses if we identify materially equivalent propositions: if $p$ and $q$ are two different propositions, then $\{p, q\}$ and $\left\{p, q, p_{\{p, q\}}\right\}$ will be two different classes associated with one and the same proposition. ${ }^{17}$ The proposition that every proposition in $\{p, q\}$ is true is, after all, materially equivalent to the proposition that every proposition in $\left\{p, q, p_{\{p, q\}}\right\}$ is true. But as Russell observed:

[...] such an escape is in reality impracticable, for it is quite self-evident that equivalent propositional functions are not identical. Who will maintain, for example, that " $x$ is an even prime other than 2" is identical with " $x$ is one of the Charles II's wise deeds or foolish sayings"? Yet those are equivalent if a well-knonw epitaph is to be credited.

Matters improve a little when we adopt a more reasonable standard for the individuation of propositions: necessary equivalence. If you think that propositions are classes of possible worlds, for example, then you will thereby identify necessarily identical propositions and $(R 2)$ will simply become false: if $p$ and $q$ are two different propositions, then $\{p, \neg p\}$ and $\{q, \neg q\}$ will be two different classes to which one and the same proposition corresponds, namely, the impossible proposition. But maybe the identification of necessarily equivalent propositions is overkill. The moral of Russell's paradox of propositions might just be that, contrary to expectations, some propositions cannot assert something of a class of propositions without thereby asserting the very same thing of a different class of propositions. This realization should ideally fall out of a reasonable account of propositions, but, in the meantime, it may simply be regarded as a logical constraint on how fine-grained we can coherently take propositions to be.

\footnotetext{
${ }^{16}$ If a proposition $q$, is not a proposition associated to any class of propositions, then $q$ is not the first component of any ordered pair in $R$ and we may think of $R$ as encoding a class-valued function on which $q$ is assigned the empty class of propositions.

${ }^{17} p_{\{p, q\}}$ is the proposition: every proposition in the class $\{p, q\}$ is true.
} 
The alternative is to reject $(R 3)$, which is an impredicative instance of a class comprehension schema. The reason $(R 3)$ is impredicative is that it involves quantification over classes of propositions. There are at least two main options at this point. One option is to restrict the schema of class comprehension to predicative conditions in which no bound class variables are allowed to occur. Russell's argument collapses once $(R 3)$ is withdrawn on account of its impredicativity. ${ }^{18}$

There is an alternative objection to (R3) recently explored in (Deutsch 2014). As John von Neumann conceived of the distinction between sets and proper classes, it was a distinction between members and non-members: unlike sets, proper classes cannot be members of other classes. (Deutsch 2014) suggests that we can profitably extend the distinction between members and nonmembers to the case of propositions. What we learn from Russell's paradox of propositions, according to (Deutsch 2014), is that, like proper classes, some propositions cannot be members. They are non-members. $(R 3)$ is false because it overlooks the fact that many propositions fail to be members. When appropriately restricted, a weaker counterpart of $(R 3)$ should state that there is a class of all and only propositions $p$ such that (i) $p$ is a member, and (ii) $p=p_{C}$ for some class of propositions $C$ such that $p \notin C$. Call this class of propositions $R^{-}$. By $(R 1)$, there is a proposition $p_{R^{-}}$ corresponding to $R^{-}$. But Russell's contradiction is averted on the assumeption that $p_{R^{-}}$can never be a member of a class. In fact, (Deutsch 2014) turns the Russellian argument into a proof that $p_{R^{-}}$ is a non-member.

This response fits well with the construal of classes as set-like objects other than sets. But given the official stance we have adopted in this paper, this solution to Russell's paradox of propositions comes at a very high cost. To speak of a class of propositions is merely to speak of them in the plural, and, likewise, to be a member of a class of propositions is merely to be one of them. So, for a proposition to be a non-member is for it to never be able to count as one of some propositions. But how could there not be some propositions of which a given proposition $p$ is one? Does $p$ itself not constitute a plurality of which $p$ itself is one?

Neither response to Russell's paradox of propositions is cost-free, but the real concern is that modern descendants of the paradox have been used to cast doubt upon the coherence of possible worlds accounts of modality of the sort mentioned in the introduction. To rehearse a version of the problem, notice that proponents of the schema $(\diamond)$ tend to speak of propositions, and while they differ as to how best to conceive of them, they often agree on two minimal constraints propositions and

\footnotetext{
${ }^{18}$ Russell's paradox of propositions is structurally analogous to Russell's derivation of a contradiction from Frege's Axiom $\mathrm{V}$ according to which every concept determines an extension to which exactly its instances belong. But it is now known that there are consistent predicative fragments of Grundgesetze, and the arguments for the consistency of Axiom $\mathrm{V}$ against the background of predicative restrictions of second-order comprehension can be transposed mutatis mutandis to the case of $(R 1)$ and $(R 2)$ against the background of predicative class comprehension. The consistency of predicative fragments of Grundgesetze has been discussed in (Parsons 1987), (Heck 1996), and (Ferreira and Wehmeier 2002).
} 
worlds ought to satisfy:

(P1) Each proposition $p$ has a negation $\neg p$.

( $P 2$ ) If $p$ is a proposition and $w$ is a possible world, then either $p$ is true at $w$ or $\neg p$ is true at $w$.

Call a class $C$ of propositions maximal if, and only if, for each proposition $p$, either $p$ is a member of $C$ or $\neg p$ is a member of $C$. And call a class $C$ of propositions compossible if, and only if, it is possible that every proposition in $C$ is true. Given $(P 1)$ and $(P 2),(\diamond)$ seems to lead to:

(P3) If $w$ is a possible world, then the propositions that are true at $w$ form a maximal compossible class of propositions.

One is now in a position to deploy a variant of Russell's paradox of propositions in order to argue that it is incoherent to suppose that there are any maximal compossible classes of propositions. In particular, one argues for the inconsistency of four apparently plausible principles:

(M1) There is a maximal compossible class $M$ of propositions.

(M2) For each class $C$ of propositions in $M$, there is a proposition $p_{C}$ associated with $C$, e.g., the proposition that every proposition in $C$ is true.

(M3) If $C$ and $D$ are different classes of propositions in $M$, then the four propositions $p_{C}, \neg p_{C}$, $p_{D}$, and $\neg p_{D}$ are pairwise different.

(M4) There is a class of all propositions $p$ such that $p$ is either $p_{C}$ or $\neg p_{C}$ for some class $C$ of propositions in $M$.

The argument is closely related to Russell's derivation of a contradiction from his premises.

By $(M 2)$, consider the proposition $p_{R}$ associated with the class $R$, which exists by (M4). We argue that both $p_{R}$ is a member of $R$ and $p_{R}$ is not a member of $R$. First, $p_{R} \in R$, Otherwise, if $p_{R} \notin R$, then $p_{R}$ satisfies a sufficient condition for membership to $R$, whence $p_{R} \in R$. Now, that we know that $p_{R} \in R$, by definition of $R$, there is some class $C$ of propositions in $M$ such that $p_{R}$ is either $p_{C}$ or $\neg p_{C}$ but $p_{R}$ is not a member of $C$. But since $R$ and $C$ differ by at least one member, namely, $p_{R}$, by $(M 3), p_{R}$ is different from $p_{C}$ and $\neg p_{C}$. Contradiction.

One may now take this argument to provide us with a reductio of $(M 1)$, which would spell trouble for any possible worlds account of modality on which every world determines a maximal compossible class of propositions.

Now, there is no question that the argument is a reductio of something, but it's hard to see what exactly. (Deutsch 2014) suggests it is a reductio of the claim that every proposition is a member, 
whereas we have suggested it is a reductio of a very fine-grained conception of proposition. Suffice it to say, for present purposes, that the argument will be powerless against a coarse-grained conception of propositions on which one identifies necessarily equivalent propositions. If necessary equivalence is sufficient for propositional identity, then there is prima facie no motivation whatever for the likes of (M3) and the difficulty is avoided. And even if more fine-grained conceptions of propositions are wanted, you may still take the problem to provide us with a logical constraint on granularity: propositions cannot be as fine-grained as to validate the likes of (M3).

\subsection{Kaplan's Paradox}

(Kaplan 1995) observed that (A) is unsatisfiable against the background of a possible worlds model theory for intensional logic:

(A) $\forall p \diamond(Q p \wedge \forall q(Q q \leftrightarrow(p=q)))$.

If, like Kaplan, we interpret the sentential operator, $Q p$, to mean: "it is wondered whether it is the case that $p$ " or " $p$ is queried" for short, then (A) states that for each proposition $p$, it could be the case that $p$, and $p$ alone, is queried. Kaplan himself conceived of it as a difficulty for the standard possible worlds model theory for intensional logic: (A) is unsatisfiable, when it should not be ruled out by logic alone. A model generally interprets propositional quantification in terms of objectual quantification over sets of possible worlds in the model. Since $\diamond B$ is true at a world in the model just in case $B$ is true at some world in the model, the satisfiability of $(A)$ would require, for each set of possible worlds, a world to bear witness to the possibility that the corresponding proposition, and it alone, be in the extension of $Q$ at a world. And this in turn is inconsistent with Cantor's theorem according to which there are strictly more sets of possible worlds than there are possible worlds in the model. ${ }^{19}$

Kaplan's observation has often been turned into a cardinality problem for $(\diamond)$. If the possibility that a given proposition $p$, and $p$ alone, is queried requires a possible world at which $p$, and $p$ alone, is queried, then (A) contravenes Cantor's theorem. If possible worlds form a set, then they will have

\footnotetext{
${ }^{19}$ (Deutsch 2014) offers a different take. One route to the unsatisfiability of $(A)$ looks at the class of possible worlds $K=\{w: \exists p(w \models \forall q(Q q \leftrightarrow(p=q)) \wedge w \not p)\}$. Now, let $p_{K}$ be a propositions, which is true at all and only worlds in $K$, e.g., $\forall w\left(w \vDash p_{K} \leftrightarrow w \in K\right)$. By $(A)$, there must be some possible world $w$ such that $w \vDash \forall q\left(Q q \leftrightarrow\left(p_{K}=q\right)\right)$. But $w \vDash p_{K}$ only if $w \in K$, which means that $w \notin p_{K}$. Contradiction. We can now incorporate a distinction between members and non-members. At most, we are entitled to the class $K^{-}=\{w: w$ is a member $\wedge \exists p(w \vDash \forall q(Q q \leftrightarrow(p=q)) \wedge w \not p)\}$. We may still consider the proposition $p_{K}^{-}$, which is true at all and only worlds in $K^{-}$. Now, there is no possible world $w$ such that $w \vDash \forall q\left(Q q \leftrightarrow\left(p_{K}=q\right)\right)$. For such a world $w$, if it existed, could not be a member. But if $w$ is not a member, it is not, in particular, not a value of a function from propositions into truth values. So, it is not a world at which a proposition can be true.
} 
a certain cardinality $\kappa$, and we may then argue:

(K1) There are exactly $\kappa$ possible worlds.

(K2) For each set of possible worlds $S$, there is at least one proposition $p$, which is true at exactly the worlds in $S$.

(K3) There are at least $2^{\kappa}$ propositions, and $2^{\kappa}>\kappa$ by Cantor's theorem.

(K4) For each proposition $p$, it is possible that $p$, and $p$ alone, is queried at a time $t$ by $A$.

(K5) For each proposition $p$, there is a possible world at which $p$, and $p$ alone, is queried at time $t$ by $A$.

(K6) There are at least $2^{\kappa}$ possible worlds, contradicting $(K 1)$.

Three clarificatory remarks are in order. First, note that the problem doesn't require the identification of propositions with possible worlds. (K2) only requires that every set of possible worlds determine at least one proposition; matters are hardly better if we allow a set of possible worlds to determine more than one proposition.

Second, notice that $(K 4)$ comes from $(A)$, when $Q p$ is read: " $p$ is queried at $t$ by $A$," and the transition to $(K 5)$ is motivated by $(\diamond)$. The final step relies on the observation that no world can bear witness to the possibility that each of two propositions $p$ and $q$ are the only proposition being queried at time $t$ by $A$.

Third, and lastly, Kaplan's paradox is not avoided merely by denying the existence of a set of all possible worlds. The problem remains on the assumption that possible worlds form a proper class:

(L1) There is a class of possible worlds.

(L2) For each class of possible worlds $S$, there is at least one proposition $p$, which is true at exactly the worlds in $S$.

(L3) There are strictly more classes of possible worlds than possible worlds, by Bernays' theorem.

(L4) For each proposition $p$, it is possible that $p$, and $p$ alone, is queried at a time $t$ by $A$.

(L5) For each proposition $p$, there is a possible world at which $p$, and $p$ alone, is queried at time $t$ by $A$.

(L6) There are no more propositions than there are worlds, and, by $(L 2)$, there are no more classes of possible worlds than there are propositions.

(L7) There are no more classes of possible worlds than there are possible worls, contradicting (L3) 
What gives? (Lewis 1986) — which is independently committed with the existence of a set of possible worlds - denies the assumption that each set of possible worlds is eligible as the content of a mental state. If propositions are indeed identified with sets of possible worlds, then Lewis' solution is to reject $(K 5)$ and suggest instead that there are independent reasons to think that the majority of propositions are uneligible to give the content of a mental state. This is premised on his analytic functionalism in the philosophy of mind; for him, a mental state can only be said to have a certain content if it plays a certain functional role in the mental life of the agent. ${ }^{20}$ Since there is no prima facie plausibility to the assumption that there is anything like continuum many functional roles, let alone, more functional roles than there are possible worlds, there is no reason to assume that each and every proposition could correspond to one functional role or another.

But Lewis' response doesn't reach the root of the problem. Maybe the reason some sets of possible worlds are ineligible to provide the content of a mental state is that they are utterly unpatterned and heterogeneous; they are not even eligible as the set of possible worlds that provide the truth conditions for a sentence of natural language. However, we needn't resort to vastly miscellaneous sets of worlds in order to produce a counterexample to (A). For consider the following simple sentence:

( $L$ ) Every proposition queried at $t$ by $A$ is false.

If $(L)$ expresses a proposition at all, then it is a proposition $p_{L}$, which is true at a world $w$ if, and only if, every proposition queried at $t$ by $A$ is false. Now, notice that there is no prima facie obstacle for someone other than $A$ to query $p_{L}$ at a given time. She may even query $p_{L}$, and $p_{L}$ alone, at the very time $t$ mentioned in the sentence. But $A$ herself, in contrast, will never be in a position to query $p_{L}$, and $p_{L}$ alone, at $t$ :

(i) If $p_{L}$ is true, then if $p_{L}$ is queried at $t$ by $A$, then $p_{L}$ is false.

(ii) If $p_{L}$ is queried at $t$ by $A$, then $p_{L}$ is false.

(iii) If $p_{L}$ is queried at $t$ by $A$, then some proposition $q$ queried at $t$ by $A$ is true.

(iv) If $p_{L}$ is queried at $t$ by $A$, then some other proposition $q$ is queried at $t$ by $A$.

It appears to follow that $A$ can only query the proposition expressed by $(L)$ at $t$ if $A$ queries some other proposition at $t$. So, we have the makings of a counterexample to (A). And if (A) is abandoned as hopeless, then Kaplan's paradox is no longer a reason for abandoning $(\diamond)$. Kaplan himself is aware of the difficulty, but he thinks we should put the blame squarely on the assumption that there is such a proposition as $p_{L}$-and not on $(\mathrm{A})$.

\footnotetext{
${ }^{20}$ See (Lewis 1986), pp. 106-107.
} 
To rehearse the point more formally, we may begin with an observation from (Prior 1961), which is codified by a theorem of quantificational propositional logic:

$$
\text { (P) } Q \forall p(Q p \rightarrow \neg p) \rightarrow \exists q(Q q \wedge p) \wedge \exists q(Q q \wedge \neg q) .^{21}
$$

Prior's observation is perfectly general, but it becomes all the more remarkable when we interpret the sentential operator $Q$ by means of a propositional attitude such as "it is asserted", "it is wondered whether", "it is hoped that", etc. For example, if, like Prior, we interpret $Q$ to mean: "it is asserted by a Cretan", then $(P)$ says that the proposition every proposition asserted by a Cretan is false can only be asserted by a Cretan if a different proposition is asserted by a Cretan. Logic alone appears to rule out the scenario in which a Cretan asserts the proposition every proposition asserted by a Cretan is false, and nothing else.

Kaplan's paradox is only two steps removed from Prior's theorem in an appropriate intensional logic. The rule necessitation in combination with existential generalization quickly yields the inconsistency of an immediate consequence of $(\mathrm{A})$ :

$$
\left(A^{*}\right) \forall p \diamond(Q p \wedge \forall q(Q q \leftrightarrow(p \leftrightarrow q))) .^{22}
$$

This principle appears to make quite a weak demand on possibility: for each proposition $p$, it is possible that $p$, and only propositions materially equivalent to $p$, be queried. So, $(\diamond)$ no longer requires different possible world to serve as a witness for different possibilities corresponding to different propositions; one and the same world can bear witness to many such possibilities. And yet, by minimal extension of Prior's reasoning, $\left(A^{\star}\right)$ is outright inconsistent.

In response to similar worries, David Kaplan suggested a ramified vision of propositions on which they are classified into various orders in accordance to their subject matter. First, we begin with

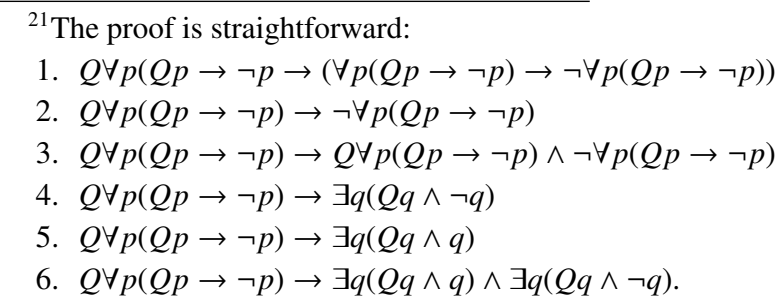

Universal instantiation From 1 by propositional logic From 2 by propositional logic From 3 by existential generalization From 2 by quantificational logic From 4 and 5 by propositional logic

${ }^{22}$ The rule of necessitation allows one to move from a theorem $B$ to its necessitation: $\square B$. Here is the argument:

1. $Q \forall p(Q p \rightarrow \neg p) \rightarrow \exists q(Q q \wedge q) \wedge \exists q(Q q \wedge \neg q)$

Prior's Theorem

2. $Q \forall p(Q p \rightarrow \neg p) \rightarrow \exists q(Q q \wedge \neg(\forall p(Q p \rightarrow \neg p) \leftrightarrow q))$

3. $\square(Q \forall p(Q p \rightarrow \neg p) \rightarrow \exists q(Q q \wedge \neg(\forall p(Q p \rightarrow \neg p) \leftrightarrow q)))$

4. $\exists p \square(Q p \rightarrow \exists q(Q q \wedge \neg(p \leftrightarrow q))$

5. $\neg \forall p \diamond(Q p \wedge \forall q(Q q \rightarrow(p \leftrightarrow q)))$

From 1 by quantificational logic

From 2 by necessitation

From 3 by existential generalization From 4 by quantificational logic 
propositions of level 0 , which are concerned with purely extensional matters like, to borrow Kaplan's illustration, distributions of earth, wind, fire, and water. Then we form propositions of level 1 , which are concerned with purely extensional matters and propositions of level 1 . Then we form propositions of level 2, etc. Once one opts for ramification, we find that a propositional variable of a given level may only range over propositions of a lower level, and one should find appropriate replacements for axioms like universal instantiation. ${ }^{23}$

Ramification gives Kaplan the resources to block both Prior's theorem and the alleged inconsistency of $\left(A^{*}\right)$, but it is not without costs. To mention just one, ramification bars absolute generalizations over propositions; when one asserts, for example, that every proposition is true or false, one should not take the very proposition being asserted to lie in the range of the universal propositional quantifier. So, logical generalizations must have appropriately restricted scope.

Given the choice between $(A)$ and the severe expressive limitations incurred by ramification, one may well take the moral of Kaplan's paradox to be that an apparently plausible principle is, on reflection, not only false but simply inconsistent. Once $(A)$ ceases to exert any pull on us, we may eventually come to terms with Kaplan's observation.

\section{Paradox and possibilia}

We now shift focus to accounts according to which whatever could exist does, in fact, exist. I utterly lack the expertise to assemble the parts of a motorcycle, and it is safe to assume that no motorcycle has ever been, or will ever be, built by me. However, had I had the expertise, I could have assembled various materials into a motorcycle. Since there could have a motorcycle assembled by me, there is, in fact, a possible motorcycle assembled by me. The thought that we should admit such merely possible objects into our ontology turns out to be prone to paradox.

David Lewis and Timothy Williamson are two philosophers, who have defended variants of the thought in print. According to (Lewis 1986), when we quantify unrestrictedly over all that exists, we quantify over merely possible objects as well as actual ones. For Lewis, to claim that a motorcycle could have been built by me is to claim that a concrete motorcyle is built by a counterpart of mine at a concrete universe causally and spatiotemporally isolated from our universe. This concrete motorcycle lies in the range of the unrestricted quantifer, which justifies the claim that there is a possible motorcycle built by me.

\footnotetext{
${ }^{23}$ One is, for example, barred from making the inference from $\forall p(Q p \rightarrow \neg p)$ to $Q \forall p(Q p \rightarrow \neg p) \rightarrow \forall p(Q p \rightarrow \neg p)$ on the grounds that the initial propositional quantifier in $\forall p(Q p \rightarrow \neg p)$ is intended to range over propositions of a strictly lower level than the proposition expressed by $\forall p(Q p \rightarrow \neg p)$.
} 
Timothy Williamson is independently committed to the view that whatever could exist does, in fact, exist. His reasons, however, are different: he endorses the universal generalization of the Barcan Formula of quantified modal logic:

$$
\diamond \exists x B \rightarrow \exists x \diamond B
$$

Since it is possible that something is motorcycle assembled by me, something is, in fact, possibly a motorcycle by me. Unlike Lewis, however, Williamson distances himself from the further claim that a merely possible motorcycle built by me is a concrete motorcycle made out of suitable metal parts and pieces; a merely possible motorcycle is not even a motorcyle, but rather a non-concrete object, which could have nevertheless been a motorcycle.

\subsection{Recombination and paradox}

One difference between modal realism and other possible worlds accounts of modality lies in Lewis' ambition to provide a reductive account of modality: in particular, his account of possible worlds is meant to remain free from any modal idioms by reducing talk of possible worlds to talk of spatiotemporally and causally isolated concrete universes. But whether or not the identification helps vindicate $(\diamond)$, will require the assurance that the domain of possible worlds is sufficiently rich and varied to ground all possibilities.

The principle of recombination, inspired by the Humean dictum that there are no necessary connections between distinct existences, is supposed to provide such an assurance. A first pass at the principle is the claim that anything can co-exist with anything else, and, likewise, anything can fail to co-exist with anything else. ${ }^{24}$ But since, according to Lewis, individuals are world-bound, contrary to this crude attempt at recombination, individuals drawn from different worlds never co-exist at any world. To deal with this obstacle, Lewis recommends a formulation of recombination in terms of duplication:

Unrestricted Recombination: Given some individuals, there is a world that contains any number of duplicates of any of them.

(Forrest and Armstrong 1984) raises a challenge for a certain form of recombination on the grounds that it is inconsistent with the existence of a set of possible worlds, and, a fortiori, a set of possibilia. Since possible worlds are objects, every world in the set of possible worlds is subject to recombination. So, they consider a possible world which non-overlapping copies each of the original worlds as parts. They later take such a world to be strictly larger in size than itself, which is absurd. Their target, however, appears to be the following principle:

\footnotetext{
${ }^{24}$ This is Lewis's initial gloss of the thought behind recombination in (Lewis 1986).
} 
Unrestricted Recombination without Overlap: Given some individuals, there is a world that contains any number of non-overlapping duplicates of any of them.

Their argument amounts to this:

(F1) No matter what some possible worlds may be, there is a world which contains non-overlapping duplicates of each of them.

(F2) There is a world, which is strictly larger in size than itself.

The existence of a world containing non-overlapping duplicates of each world, they think, leads to $(F 2)$, which is absurd. But this last step requires subsidiary argument. Let World be a world which contains a duplicate of each world in the set of all worlds:

(i) The set $E$ of electrons in World has cardinality $\kappa$.

(ii) For each non-empty subset of $E$, there is a possible world at which exactly them enjoy a certain quality $Q$.

(iii) Since there are exactly $2^{\kappa-1}$ non-empty subsets of $E$, there are at least $2^{\kappa-1}$ electrons in World, which contradicts (i) above.

The last step in the argument is justified by the fact that the relevant duplicates of the possible worlds generated by each non-empty subset of $E$ do not overlap, and they each contain at least one electron, which is part of World.

Unfortunately, as (Nolan 1996) observes, (ii) is objectionable as it stands: at most, we are entitled to the claim that for each non-empty subset of $E$ there is a world at which exactly duplicates of them enjoy a certain quality $Q$. But once we shift the focus to such worlds, there is no longer reason to think that there should be at least one possible world for each subset $D$ of $E$. One possible world $w$ could bear witness to the possibility that exactly a duplicate of $e$ enjoys $Q$, for each $e$; for the only such electron at $w$ could itself be a duplicate of each and every electron in World. So, one world will do for all singleton subsets of $E$, one world will do for all doubleton subsets of $E$, etc. So, there is still no reason to expect more possible worlds than electrons in World.

Even if the Forrest-Armstrong argument fails to refute the existence of a set of possible worlds, there is still a simple argument from Unrestricted Recombination to the conclusion that there is no set of possible objects. The argument, given in (Nolan 1996), makes use of the following consequence of unrestricted recombination:

$(N)$ For each cardinal $\kappa$ and for each object, there is a world with at least $\kappa$ duplicates of it.

The key now is to notice that $(N)$ is incompatible with the existence of a set of possible objects: 
If there is a set $S$ of all possibilia, then some cardinal $\kappa$ is the cardinality of $S$. By Cantor's theorem, $\kappa<2^{\kappa}$, but, by $(N)$, there is a possible world with $2^{\kappa}$ duplicates of a given object. Since they all are possible objects, recombination requires the set $S$ of possibilia to have cardinality $2^{\kappa}>\kappa$, which contradicts the initial assumption that $S$ has cardinality $\kappa$.

One may perhaps hope to resist $(N)$ by falling back to Lewis' qualified version of recombination:

Recombination: No matter what some objects may be, there is a world that contains any number of duplicates of any of them size and shape permitting.

The point of the qualification is to make sure recombination does not generate unwanted possibilities as to the size and structure of spacetime. As Lewis conceives of the principle, recombination is concerned with the question of what objects can exist quite apart from the question of what are the constraints on a possible spacetime. The qualification is not very informative; there is no reason to think spatiotemporal manifolds could not include more than four, or seven, or finitely many dimensions. The hope, however, is that there is some restriction on the size of possible spatiotemporal manifolds, which can nevertheless accommodate all the possibilities $(\diamond)$ demands.

To the extent to which $(N)$ is not an immediate consequence of Recombination, one may hope to fend off the commitment to a proper class of possible objects. On the other hand, nothing in Lewis' statement of recombination precludes the truth of similar principles according to which, for each cardinal $\kappa$, there could be at least $\kappa$ objects of kind $K .^{25}$ Alternatively, one could follow (Menzel 2014) and make changes elsewhere in the set-theoretic framework and making allowance for the existence of "wide sets" that are too large to be measured by a cardinal number.

More recently, (Hawthorne and Uzquiano 2011) have argued that the likes of $(N)$ threaten to raise a more serious challenge for modal realism when combined with plausible mereological assumptions. Call a kind $K$ disperse if, and only if, a fusion of objects of kind $K$ is identical to a fusion of some objects of kind $K$ only if the former objects of kind $K$ are the same as the latter; otherwise, call the kind non-disperse. The kind portion of clay, for example, is non-disperse because a fusion of two portions of clay may be identical, for example, to a fusion of four portions of clay, each of which are proper parts of the original two portions. On the other hand, the kind person may reasonably be assumed to be disperse, since a fusion of persons will be identical to the fusion of some persons if and only if the former persons are the same as the latter.

(Hawthorne and Uzquiano 2011) are concerned with principles like this:

$(H)$ For each cardinal $\kappa$, there is a world with at least $\kappa$ angels.

\footnotetext{
${ }^{25}$ One way to see this is by noting that nothing in Recombination rules out the possibility of co-location.
} 
The possibility of angel co-habitation makes $(H)$ plausible even in the face of unexpected constraints on the possible cardinality of the set of spacetime regions. ${ }^{26}$

However, like $(N)$ before, this principle delivers a proper class of angels, many of which may be only merely possible. This, by itself, should not be cause for alarm, since we have made allowance for possible objects to form a proper class. Rather, the problem with $(H)$ is that it seems incompatible with two reasonable assumptions:

Unrestricted Composition: No matter what some objects may be, there is a fusion of them.

Maximality: All proper classes are equinumerous.

Unrestricted Composition, for Lewis, allows one to fuse objects from different worlds. But, since the kind angel is disperse, we know that different classes of possible angels have different fusions, which, in turn, tells us that there are at least as many fusions of possible angels as there are classes of possible angels. By Bernays' theorem, we know that there are strictly more classes of possible angels than there are possible angels, which means that there are strictly more fusions of possible angels than there are possible angels. Unfortunately, we already had a proper class of possible angels, which means that the class of fusions of possible angels is strictly larger in size than the class of possible angels, contradicting Maximality.

In the face of this, it looks like we must reject $(H)$ as well as similar principles for other disperse kinds: if $K$ is non-modular, then there is some cardinal $\kappa$, we know not which, such that it is just not possible for there to be $\kappa$ objects of kind $K$.

Some of the problems with recombination have been specific to Lewis' framework, but the last two issues are quite independent from the specific details of modal realism and generalize to a metaphysics of modality in which we allow for quantification over merely possible objects. In particular, they generalize to the modal metaphysics espoused by (Williamson 2013). Let us consider the first problem first. (Sider 2009) has explained how to generalize the difficulty in Williamson's framework. There is, of course, no reason to think that Williamson would accept $(N)$ as stated, since it concerns the existence of a possible world with duplicates of a given object. There is, however, a different plausible principle with similar consequences:

$\left(N^{\diamond}\right)$ For each cardinal $\kappa$, it is possible that at least $\kappa$ individuals exist.

Remember that the thrust of the Barcan Formula is that what can exist does, in fact, exist; if it is possible for there to be an individual, then there is a possible individual; by the ncessity of

\footnotetext{
${ }^{26}$ There is nothing special about angels. Certain kinds of elementary particles would have done just as well; bosons, for example, are elementary particles with integral spin, which are generally thought to be able to co-habit a point-sized region.
} 
distinctness, if it is possible for there to be two different individuals, then there are two different individuals; and, more generally, if it is possible for there to be $\kappa$ different individuals, then there are at least $\kappa$ different possible individuals. ${ }^{27}$ Since $\left(N^{\diamond}\right)$ tells us that there is no upper bound on the possible cardinality of individuals, there is no upper bound on the cardinality of possible individuals. It follows that there is no set of all possible individuals; they form a proper class.

For a generalization of the second problem, consider the principle:

$\left(H^{\diamond}\right)$ For each cardinal $\kappa$, it is possible that at least $\kappa$ angels exist.

An appropriate generalization of the Barcan Formula delivers again a proper class of possible angels. But now, if the kind possible angel is disperse, then there are strictly more fusions of possible angels that there are merely possible angels. This, like before, contravenes Maximality, since we would seem to have specified two different proper classes that are not equinumerous.

One way out, of course, is to confine composition to avoid the commitment to fusions of merely possible angels. Since, unlike Lewis, Williamson takes merely possible objects to be non-concrete, one might restrict composition to the realm of the concrete. On this view, we would have a principled reason to resist the existence of strictly more fusions of possible angels than possible angels. ${ }^{28}$ Still, there is no denying that this comes at a cost.

\section{Conclusion}

Modal paradox threatens possible worlds accounts of modality, but they are far from decisive. Modern descendants of Russell's paradox of propositions can be resisted, provided one takes propositions to be sufficiently coarse-grained as to be in a position to resist that different classes of propositions must be associated with different propositions; some propositions can only assert something of a class of propositions if they assert the same thing of a different class of propositions.

Kaplan's paradox presents a powerful challenge of its own, but it need not lead to the rejection of $(\diamond)$, provided one is prepared to relinquish $(A)$ in the face of its inconsistency. The fact that logic alone appears to require the rejection of a substantially weaker principle is further evidence against $(A)$; moreover, the very inconsistency of $(A)$ suggests that the problem does not even require to analyze possibility in terms of truth at one possible world or another. The problem persists for philosophers, who reject $(\diamond)$ and nevertheless find $(A)$ attractive.

We have surveyed, in addition, the threat of paradox for the thought that whatever could exist does,

\footnotetext{
${ }^{27}$ See (Sider 2009) for a rigurous regimentation of the argument.

${ }^{28}$ (Hawthorne and Uzquiano 2011) discusses some other responses as well.
} 
in fact, exist as it arises for two different variants of the view. We found that one is soon led to the existence of a proper class of merely possible worlds, which turns out to be in tension with Unrestricted Composition and Maximality. Though none of the problems constitutes a decisive refutation of the thought, they all seem to require some uncomfortable choices.

\section{References}

Adams, Robert Merrihew (1974). "Theories of Actuality”. In: Noûs 8.3, pp. 211-231.

Barwise, Jon (1975). Admissible Sets and Structures: An Approach to Definability Theory. SpringerVerlag.

Bernays, Paul (1942). "A system of axiomatic set theory: Part IV. general set theory". In: The Journal of Symbolic Logic 7.4, pp. 133-145.

Cantini, Andrea (2004). "On a Russellian paradox about propositions and truth". In: One Hundred Years of Russell's Paradox, pp. 259-84.

Cocchiarella, Nino B (2000). "Russell's paradox of the totality of propositions". In: Nordic Journal of Philosophical Logic 5.1, pp. 25-37.

deRosset, Louis (2009a). “Possible Worlds I: Modal Realism”. In: Philosophy Compass 4.6, pp. 9981008 .

— (2009b). "Possible Worlds II: Non-Reductive Theories of Possible Worlds". In: Philosophy Compass 4.6, pp. 1009-1021.

Deutsch, Harry (Jan. 2014). "Resolution of some paradoxes of propositions". In: Analysis 74.1, pp. 26-34.

Ferreira, Fernando and Kai F. Wehmeier (2002). "On the Consistency of the $\Delta_{1}^{1}$-CA Fragment of Frege's Grundgesetze”. In: Journal of Philosophical Logic 31.4, pp. 301-311.

Forrest, Peter and D. M. Armstrong (1984). "An Argument Against David Lewis' Theory of Possible Worlds". In: Australasian Journal of Philosophy 62.2, pp. 164-168.

Friedman, Harvey. Faithful Representation in Set Theory with Atoms.

Hawthorne, J. and G. Uzquiano (2011). "How Many Angels Can Dance on the Point of a Needle?

Transcendental Theology Meets Modal Metaphysics”. In: Mind 120.477, pp. 53-81.

Heck, Richard (1996). “The Consistency of Predicative Fragments of Frege's Grundgesetze der Arithmetik”. In: History and Philosophy of Logic 17.1, pp. 209-220.

Kaplan, David (1995). “A Problem in Possible-World Semantics”. In: Modality, Morality and Belief: Essays in Honor of Ruth Barcan Marcus. Cambridge: Cambridge University Press, pp. 4152.

Kripke, Saul A. (1980). Naming and Necessity. Harvard University Press. Lewis, David K. (1986). On the Plurality of Worlds. Blackwell Publishers. 
Maddy, Penelope (1983). "Proper Classes". In: Journal of Symbolic Logic 48.1, pp. 113-139. Menzel, Christopher (2014). "Wide Sets, ZFCU, and the Iterative Conception". In: Journal of Philosophy 111.2, pp. 57-83.

Nolan, Daniel (1996). "Recombination Unbound". In: Philosophical Studies 84.2-3, pp. 239-262. Oksanen, Mika (1999). "The Russell-Kaplan paradox and other modal paradoxes: a new solution". In: Nordic Journal of Philosophical Logic 4, pp. 73-93.

Parsons, Terence (1987). "On the Consistency of the First-Order Portion of Frege's Logical System”. In: Notre Dame Journal of Formal Logic 28.1, pp. 161-168.

Plantinga, Alvin (1976). “Actualism and Possible Worlds”. In: Theoria 42.1-3, pp. 139-160.

- (1992). The Nature of Necessity. Vol. 25. 99. Clarendon Press, p. 178.

Prior, Arthur (1961). "On a family of paradoxes". In: Notre Dame Journal of Formal Logic 2.1, pp. 16-32.

Quine, Willard V (1937). "New foundations for mathematical logic”. In: American mathematical monthly, pp. 70-80.

Rubin, Herman and Jean E Rubin (1985). Equivalents of the Axiom of Choice II. Amsterdan: North Holland.

Russell, Bertrand (1903). The Principles of Mathematics. W. W. Norton \& Company.

Sider, Theodore (2009). "Williamson's Many Necessary Existents". In: Analysis 69.2, pp. 250258.

Stalnaker, Robert C. (1976). "Possible Worlds". In: Noûs 10.1, pp. 65-75.

Uzquiano, Gabriel (2014). "Recombination and Paradox".

Williamson, Timothy (2013). Modal Logic as Metaphysics. Oxford University Press. 